\title{
Decision support of Bad Player Identification in MOBA Games using PageRank based Evidence Accumulation and Normal Distribution based Confidence Interval
}

\author{
Jae Youn Shim, Tae Hyun Kim and Seong Whan Kim, \\ Department of Computer Science, University of Seoul, \\ 13 Siripdae-gil, Dongdaemun-gu, Seoul 130-743, \\ simpo@uos.ac.kr,kdtth@daum.net, Corresponding Author: swkim7@uos.ac.kr \\ Abstract
}

On-line game service is the hottest game genre today, and one of major on-line game is MOBA (multiplayer online battle arena) game. In MQBA games, the collaboration of team players and team strategy are vital elements togethen besides the individual player's game control capability. Thus important issue for the MOBA service prbviders is to detect bad players showing abnormal plays or appearances in games with embedded malicious intentions. Previous approach had been presented to cope with such players; however they have not yet shown any promising results to judge each player. In this paper, we propose an efficient and automatic abnormal player decision support scheme using PageRank and normal distribution to find and judgebad players. Our scheme computes $B P R$ (bad player ranking) for each user, and BPR can be used in service provider's final decision to decide a specific usér a âh abnormál player. Our scheme has main advantage that it requires small computatidnal efforts to identify bad players, because we utilize PageRank algorithm which shows effrcient computation and information search capability.

Keywords: MOBA (multiplayế online battle arena), PageRank, Decision support system, Bad player

\section{Introduction}

The on-line game market has been growing continuously. MMORPG (massively multiplayer online role-playing game) genre and the RTS (real-time simulation) genre had been popular, now, the newly appeared MOBA (Multiplayer Online Battle Arena) games are on the stage. Especially, the 'League of Legends' from Riot Games, Inc. [1] became the new leader in e-Sport by prevailing, the big market of North America, China and Korea. 32 millions of people around the world watched the League of Legends World Championship Season 3 via on-line streaming and TV including 8.5 millions of simultaneous viewers. However, the big challenge to the rapidly grown MOBA games is the quality of the game degraded by bad players. Basically, the architecture of the MOBA game was designed to get victories through individual characters which have been grown on the team strategy and collaboration of participants. Thus players ruining the collaboration of team members or using vulgar terms should be regulated by limiting chances to join the game or imposing appropriate penalties. Service providers of such games have been proposed several approaches to the regulation but still finding bad players in games and making right judgment on them remain as a difficult problem [2].

In this paper, we propose PageRank based decision support system to identify bad players in MOBA games. The basic idea of the PageRank system says that the more important page is the page which has more links to other sites than other pages, because it was more important than other pages. We utilize the idea of PageRank, and we compute JP (judgment point) and BPP (bad player point) for each player. JP is configured by the average number of reporting and the time of game playing of the player and player's 
report time. If Players have higher BPP then their JP is decreased. Total JP of players would remain unchanged but the effectiveness of the judgment on other players based on the JP for one player would be decreased in proportion to the number of reporting of bad players.

In this paper, the MOBA game will be introduced briefly, and 'THE TRIBUNAL' which is the most important institutionalized system on stage to find and judge bad players will also be discussed. And the PageRank will also be introduced along with the Decision Support System derived therefrom. In Section 2, we introduce what are MOBA and TRIBUNAL, and review related technologies, and propose our idea and computational algorithms in Section 3. We conclude in section 4 with future research directions.

\section{Related Works}

On-line game is computer or video game using network like internet. On 4 ine gaming is a technology rather than a genre, a mechanism for connécting players together rather than a particular pattern of gameplay [3]. Massively multiplayer online gandes are possible to allow hundreds of thousands of players to play the same game together using the Internet. On-line games can be the ability to connect to multiplayer games. On-line games are availability of wide variety of games for all type of game platyers and some on-line games don't need require payment. Figure 1 shows communication of on-line game player and server.

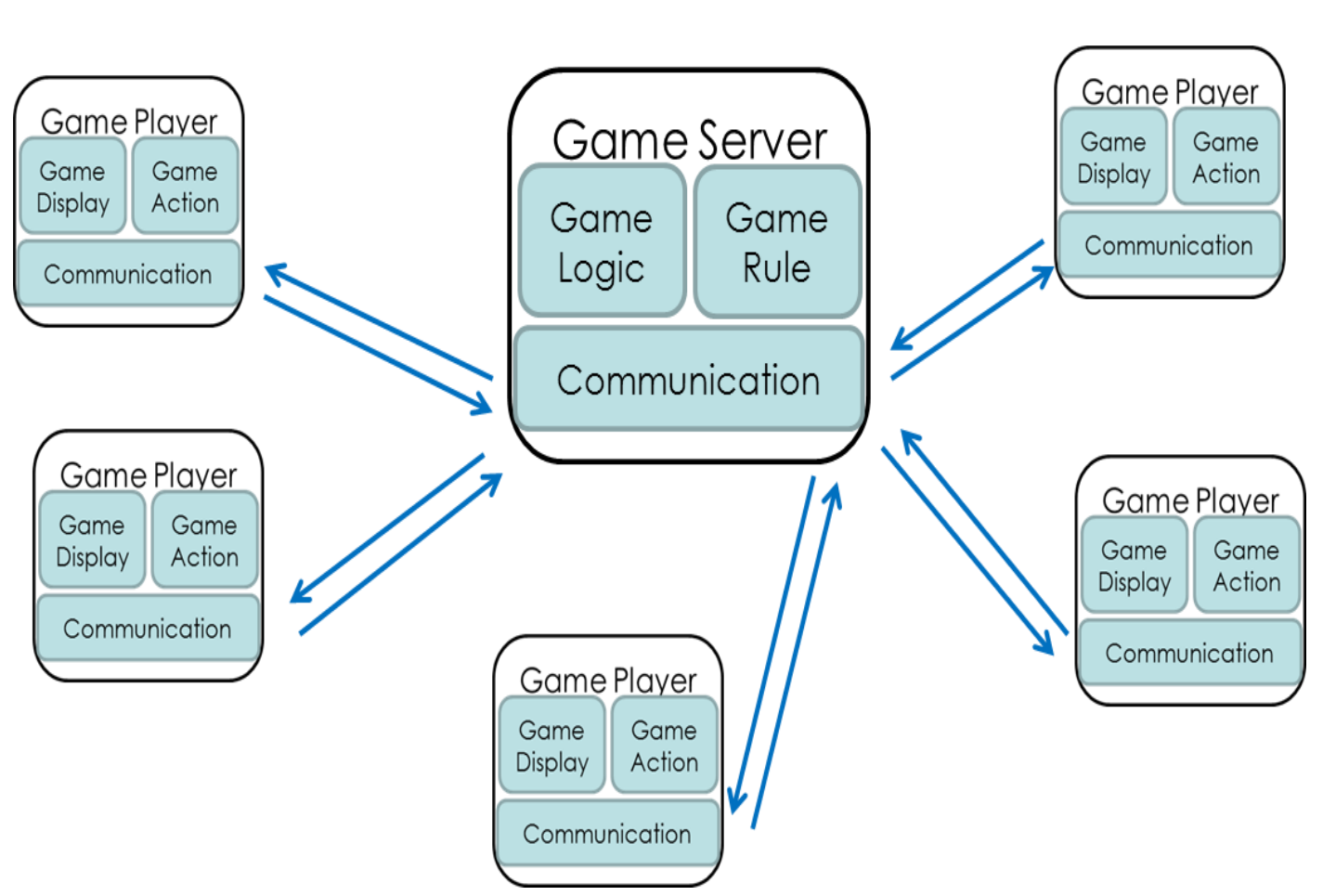

Figure 1. Communication Architecture for Online Game System

\subsection{MOBA Game and Abnormal Behavior}

The genre of online game like MOBA is currently dominating the market of online game with the growth of DOTA (defense of the ancients) [4] and LOL (league of legends) [5]. Games of MOBA are called AOS (aeon of strife) or ART (action real time strategy) which are types of the game managing Real Time Strategy originated from the Aeon of Strife, the use-map in the Star Craft. Figure 2 shows the exemplar game play screen of AOS, DOTA, and LOL. 


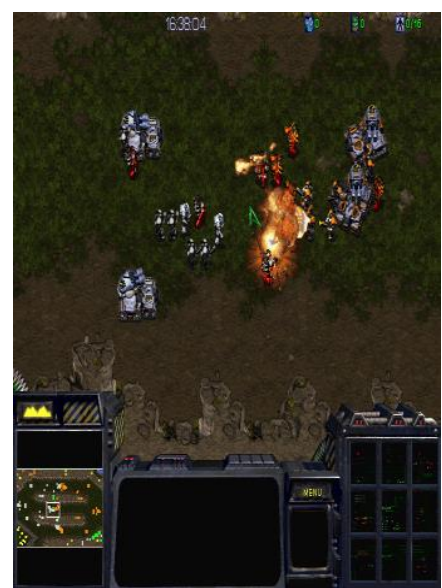

(a)

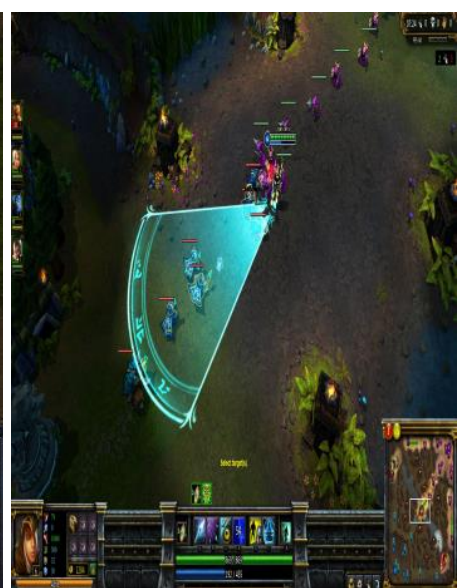

(b)

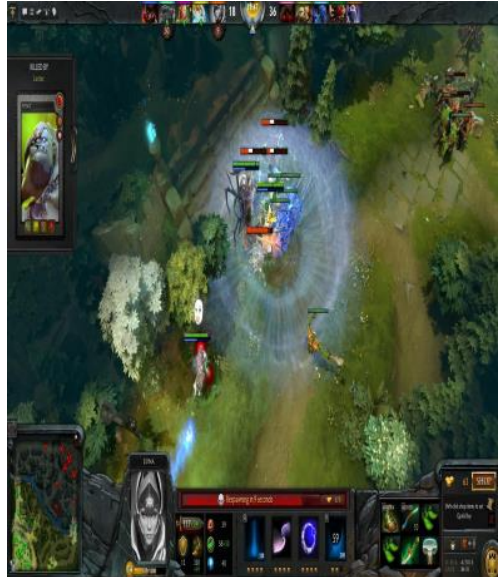

(c)

Figure 2. (a) AOS (b) LOL (c) DOTA Game Play Screen

Basic configuration the MOBA game was designed to destroy the core base of opponents by defeating opponent's players over the defenses consisted of soldiers called Minions and fortress towers by mobilizing yarious combination of selected 3 5 hero characters respectively [1]. Figure 3 shows typical formation of the map, small circles is the location of fortress towers. Neutral monsters are normalty arranged inside the triangle placed in the middle of the map to helpthe growth of heroes.

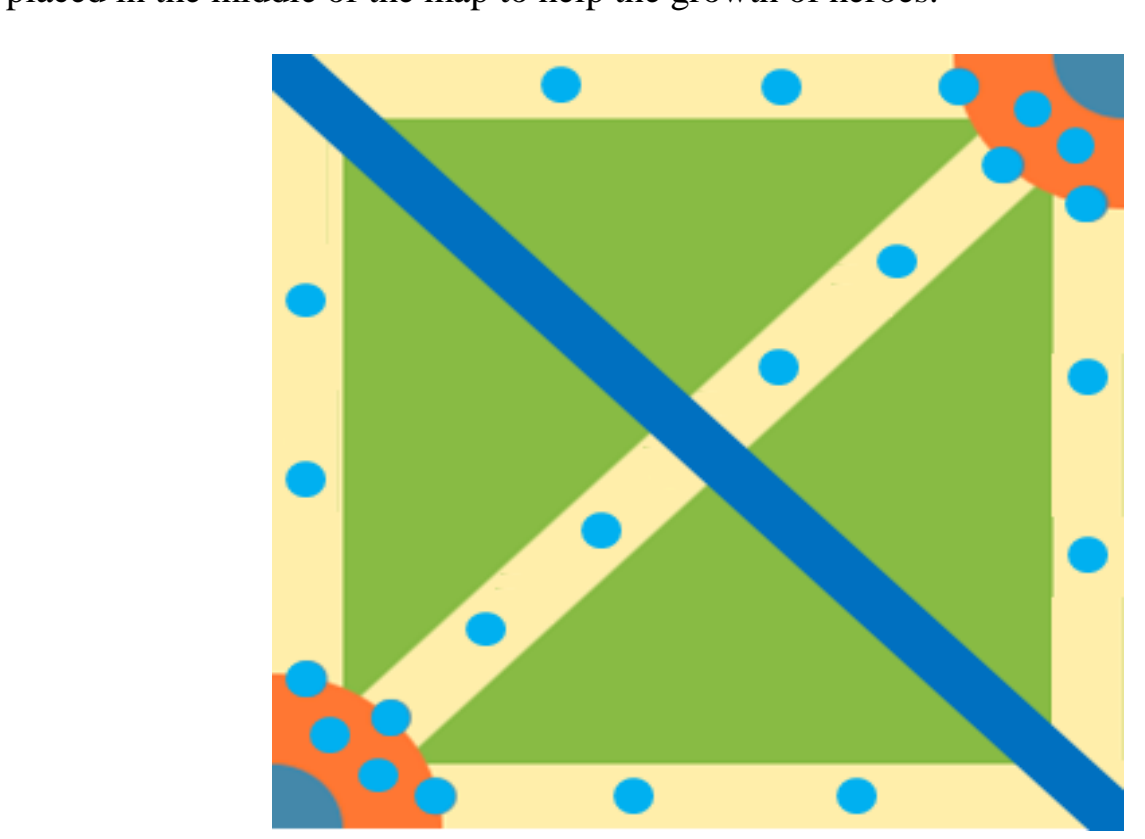

Figure 3. Typical Map of MOBA Games

Hero characters selected by players have various properties and skills and are nourished by the level-up from growing and with combination of the items. Items can be purchased with moneys created by defeating opponent minions or opponents' heroes, neutral monsters. Along with this victory, the experience marks required for the level-up also increase. The experience marks or moneys also increase with the incidents of providing aids to capture opponent heroes depending on the scheme of games and some games allow corresponding buffs for predetermined period of time when special neutral monster are captured. Experience marks or items are only applicable to each only one game. Figure 4 shows buffs monsters of LOL. 

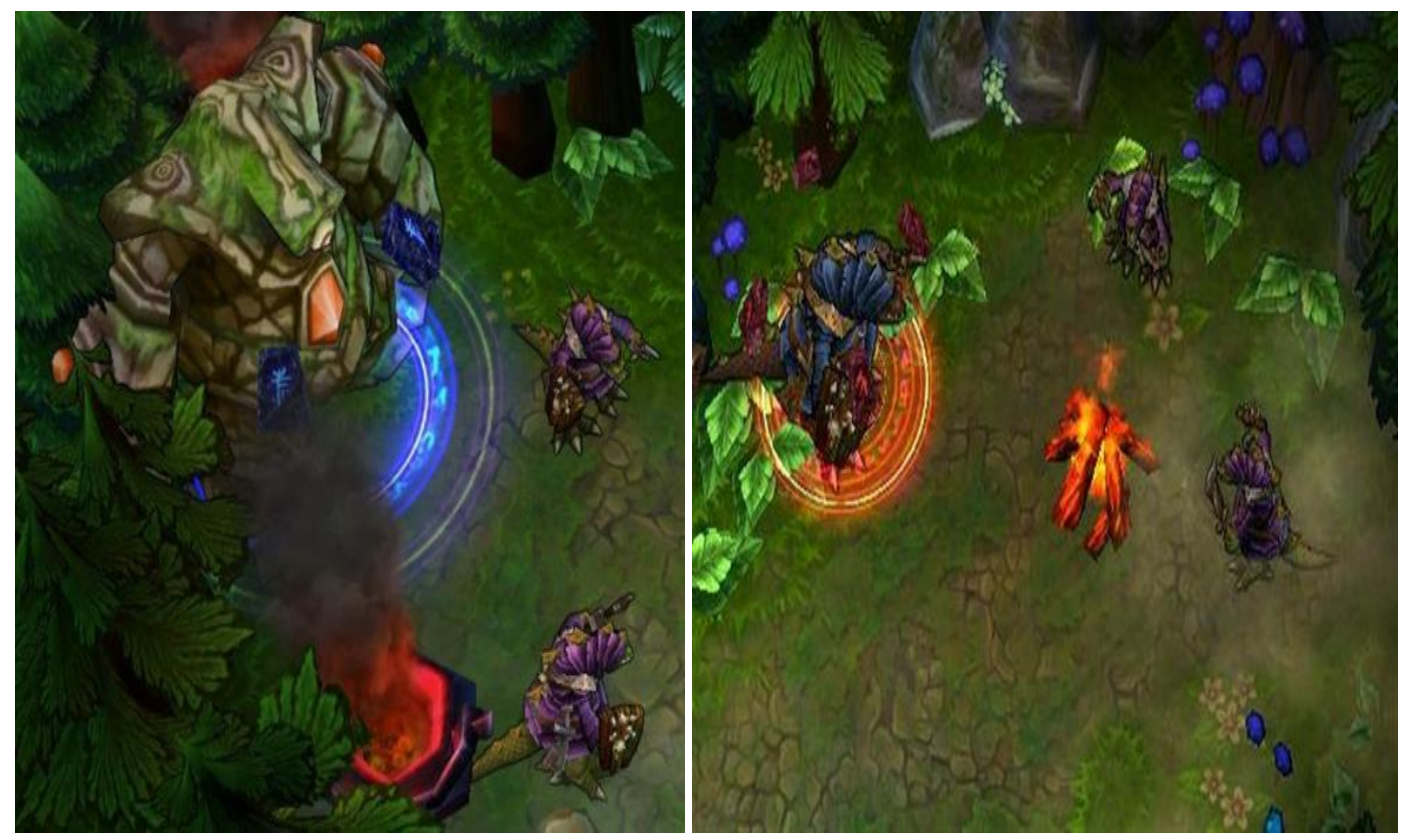

Figure 4. Buffs Monsters of LOL

There are many hero characters in the MOBA game and there can be mutuality between hero characters but the critical key to the victory of the game will be the player's control capabilities and collaboration of team players. Figure 5 shows hero characters of LOL and DOTA. They have over100 hero characters.

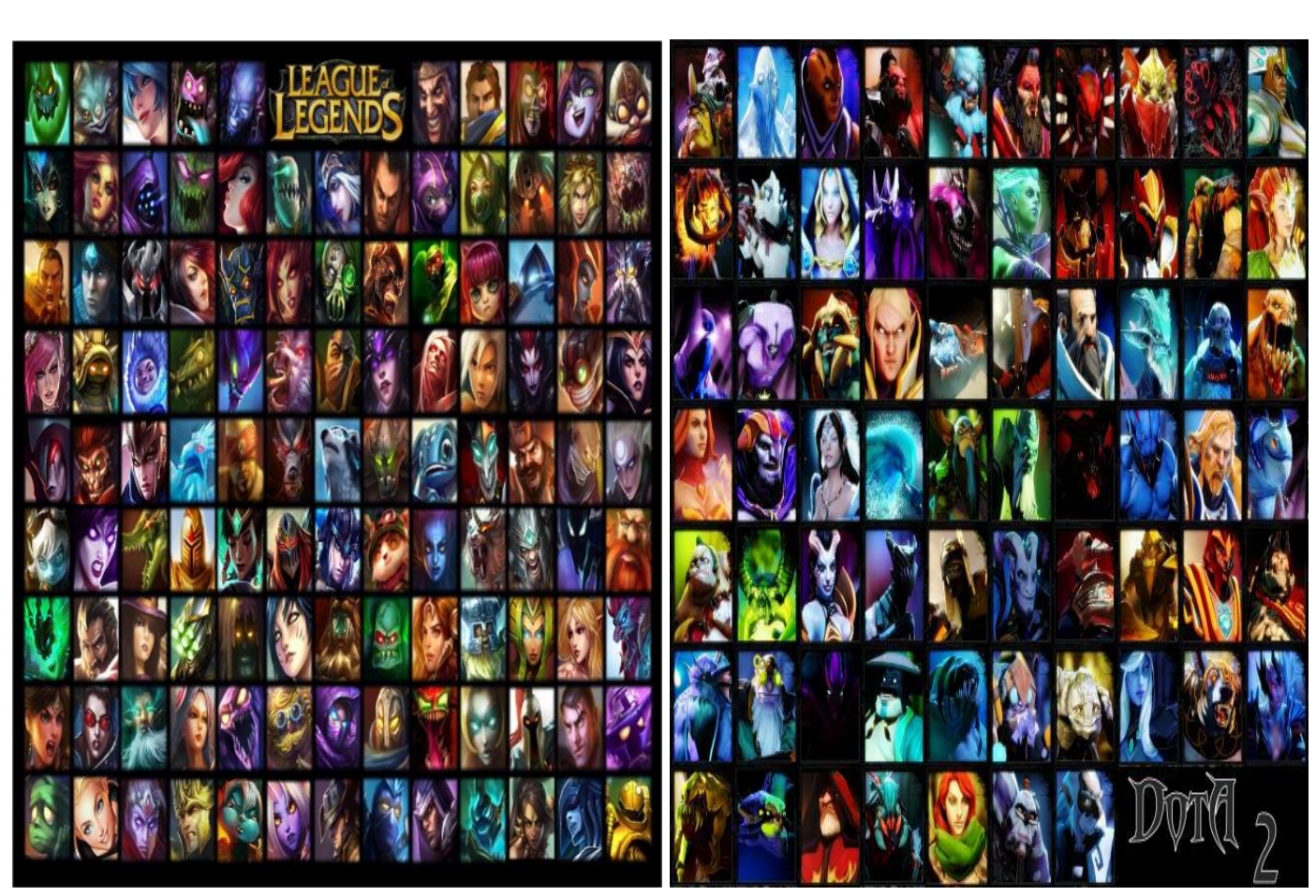

Figure 5. LOL and DOTA's Hero Characters

The advantage of the MOBA game would be the rapidity in getting the consequences compared to existing MMORPG games therefore players would not spare much on-line time to foster characters. Thus the player's proficiency of the game and control capability, and collaboration of team members will be the 
essential key to success instead of nourishing characters by playing longer times and contrary to MMORPG, player do not need to invest much time to gather moneys and items for they will be vanished when games are terminated. It is al so different from RTS games required the production of various units to solve strategic problems because the MOBA games require only one character in playing the game. In MOBA games, heroes can be configured according to various story-telling corresponded with player's preferences thus players can select characters freely. MOBA game has some bad player type like abusing, cheating. Abusing type bad game player has malicious actions or abuse. Cheating type bad game player use hack tools to win for game.

\subsection{THE TRIBUNAL: On-line Bad Player Decision Support}

Bad players participated in games like MOBA cause big trouble if they play arbitrarily ignoring team strategy or team work. The Riot Games, Inc. providing LOt adopted the 'THE TRIBUNAL' in the game to determine bad players [6]. Figure 6 shows 'THE TRIBUNAL' main page. 'THE TRIBUNAL' is an institutionalized system for players to follow the Player's rules and manners autonomously in playing the game. Players to be reported several times as bad players by other paticipants are forwarded randomly to the Tribunal where corresponding cases are configured to be judged gailty or not guilty (bad or not) by votes.

The judgment from the Tribunal is formarded to the Game Manager, and the player reported as the bad player will be put under corresponding restraint. Cases in the Tribunal usually will be configured if the number of reports exceeds certain limit. One mistake committed by chance may be reported to the Game Manager but it would not be forwarded to the Tribunal until the count of being reported as a bad player is accumulated up to a certain limit. The credit of the player who reported bad players will also be considered in the Tribunathus the reports from players who participated positively in games and reported bad players according to his/her considerate judgment will be counted for much compared to other players issuing reports recklessly.

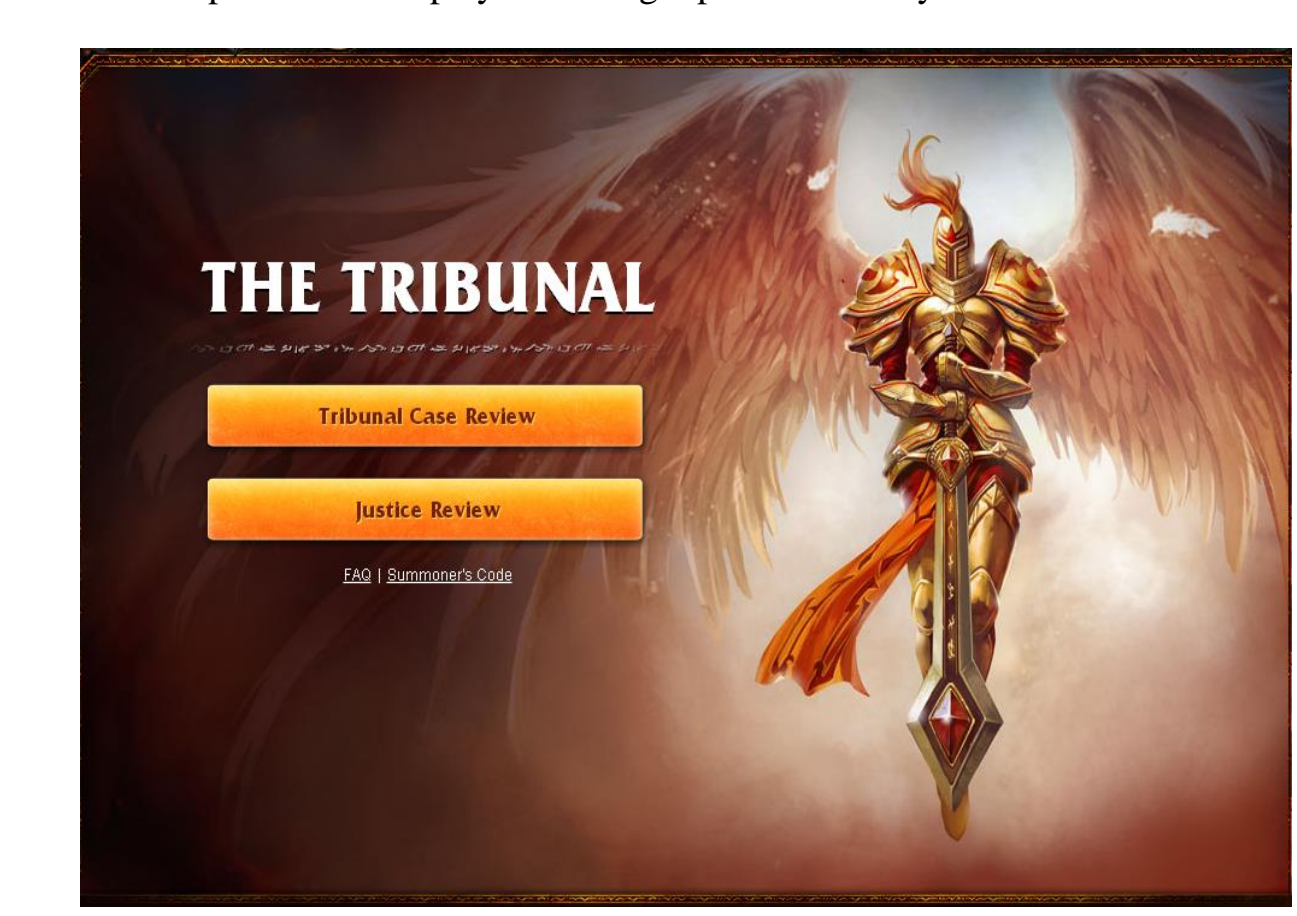

Figure 6. THE TRIBUNAL 
Generally, the good players obeying orders from the Player and playing fairly with other participants would never be forwarded to the Tribunal and cases in the Tribunal are normally configured considering the numbers of game playing. The case be reported 10 times as a bad player among thousands times of game playing would be more improbable to be forwarded to the Tribunal than the case to be reported 100 times as a bad player from 100 times of game playing, but cases committed excessive bad behaviors can be forwarded to the Tribunal regardless of the number of the game playing. So the players following general style of game playing normally would not be restrained while players creating continuous negative impacts on the game would be put under restraint. And the right scheme to make proper judgment on cases forwarded and configured by malicious intentional reports from bad players shall also be considered.

\subsection{Big Data based Approach and PageRank for Decision Support}

Big data is non-standard data set or fixed form of mass beyond the ability to be able to collect data from one database. Produced Big data system is not of traditional database system like SQL from various resources like web pages, weblog files, social media sites, e-mail, documents, game playing log. Big data can be defined with the following properties associated with it, variety data from, volume, velocity, variability, complexity, value [7]. Figure 7 shows Big data what is major feature four V. Volume is scale of data, variety is data forms, velocity is data processing speed, and veraeity is uncertainty of data.

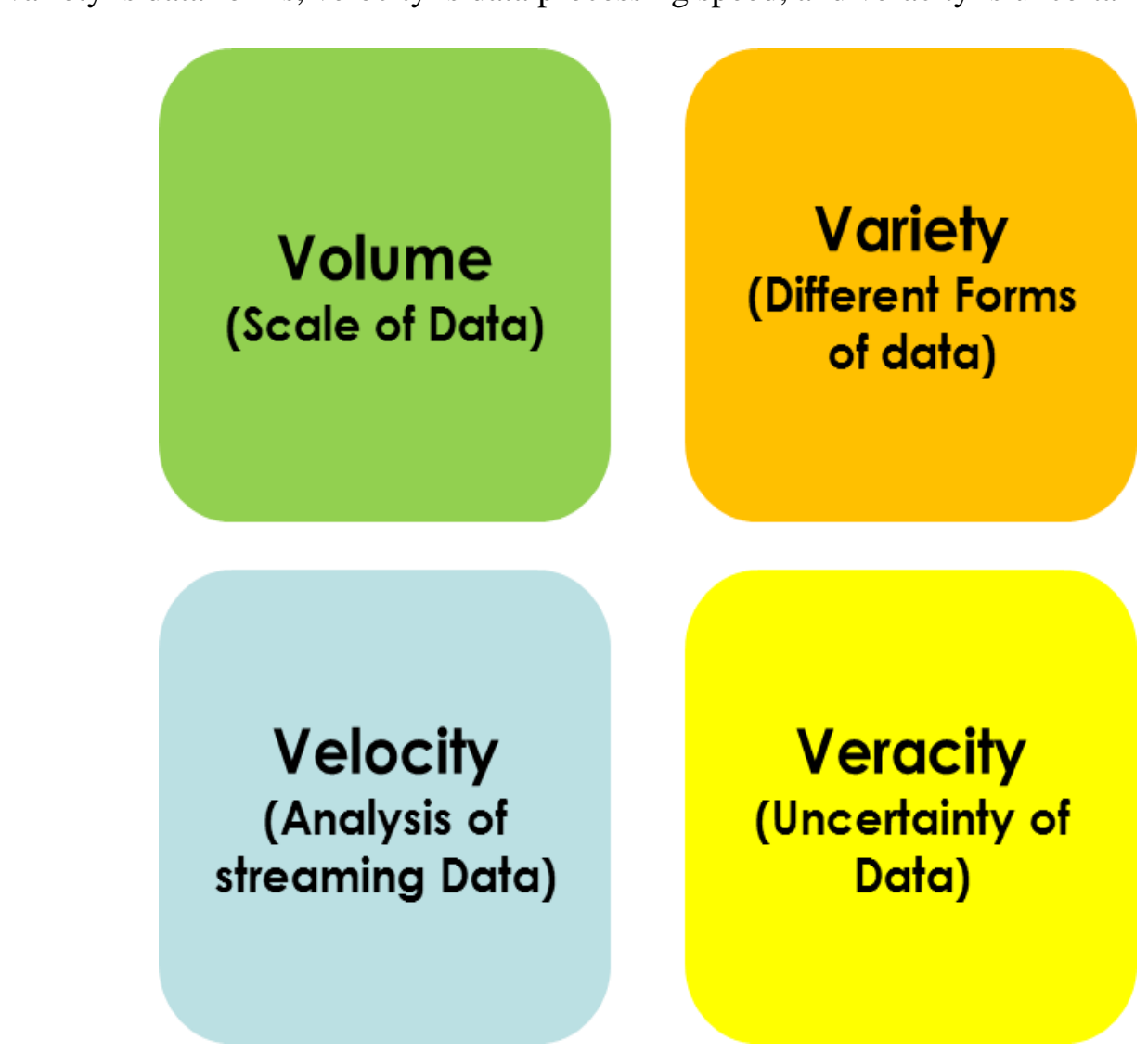

Figure 7. Four $\mathrm{V}$ of Big data

Kwon et al., discusses about the rumor propagation in online social media and how detect rumor [8]. Derive meaningful features related to the existing social and psychological theories and among features (Temporal, Structural, Linguistic) find the best set of features to discriminate rumors to non-rumor. Big data mining system needs MapReduce. Map-Reduce has two step, Map step, the master node takes the input, divides it 
into smaller sub-problems, and distributes them to worker nodes. The worker node processes the smaller problem and passes the answer back to its master node. Reduce step, the master node then collects the answers to all the sub-problems and combines them in some way to form output, the answer to problem it was originally trying to solve [9]. Figure 8 shows Map-Reduce diagram.

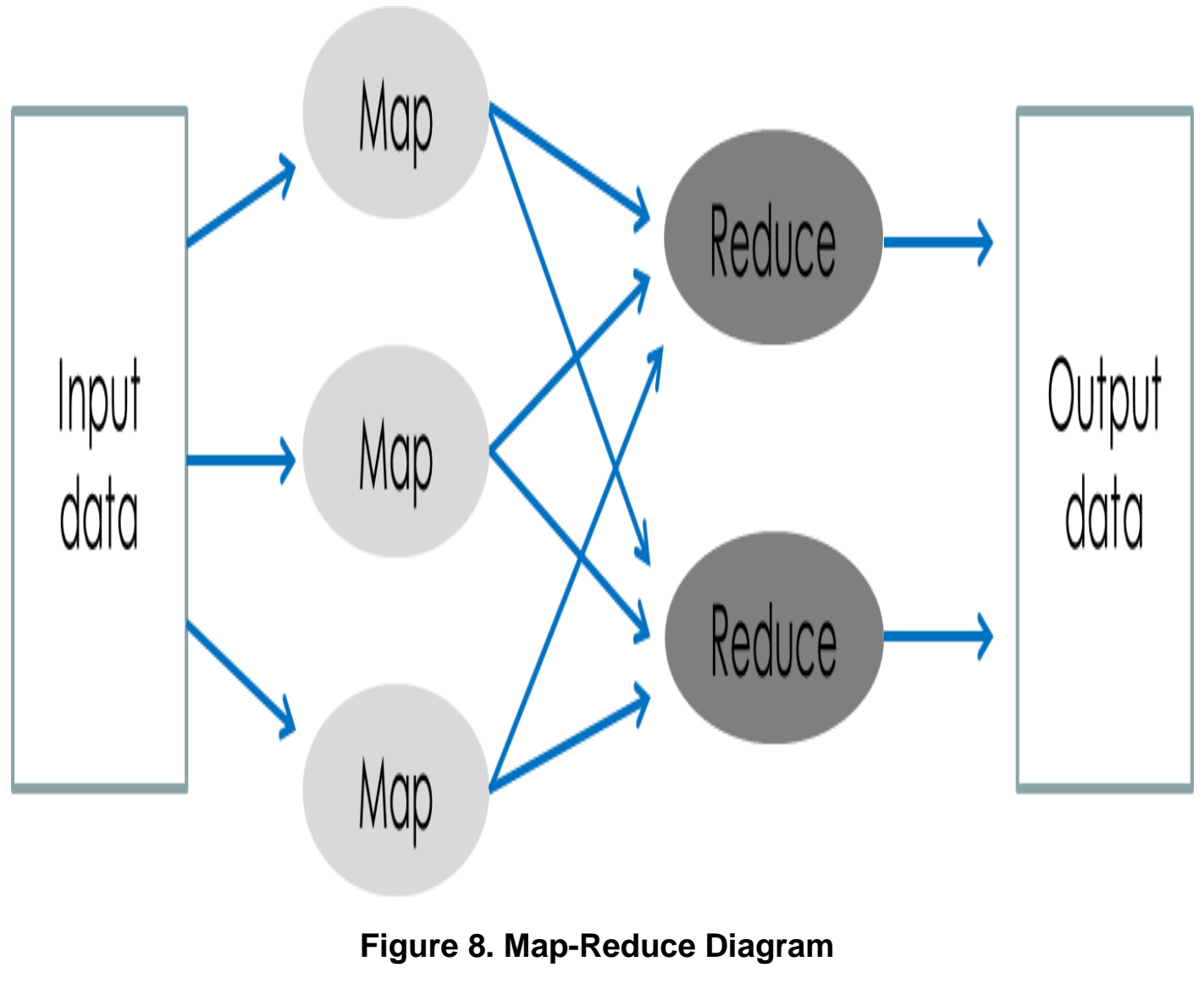

PageRank algorithm is to provide statistical inference unlike Dempster-Shafer's evidence theory, extension of the previous Bayesian inference theory [10]. The PageRank is the basic algorithm in the Google's Search Engine developed by Larry Page and Sergey Brin as a part in their study searched the new search engine [11-12]. It is an algorithm based on the observation that pages which are more important would be linked with more sites so the pages linked with important pages may have more importance than the other pages. The PageRank assumes the model which queries and searches pages called 'Random Sufer' arbitrarily and the final rank of each page is derived from a certain convergence of all weighted linked pages to respective particular rank value. The basic calculation of page rank is illustrated in Figure 9. 


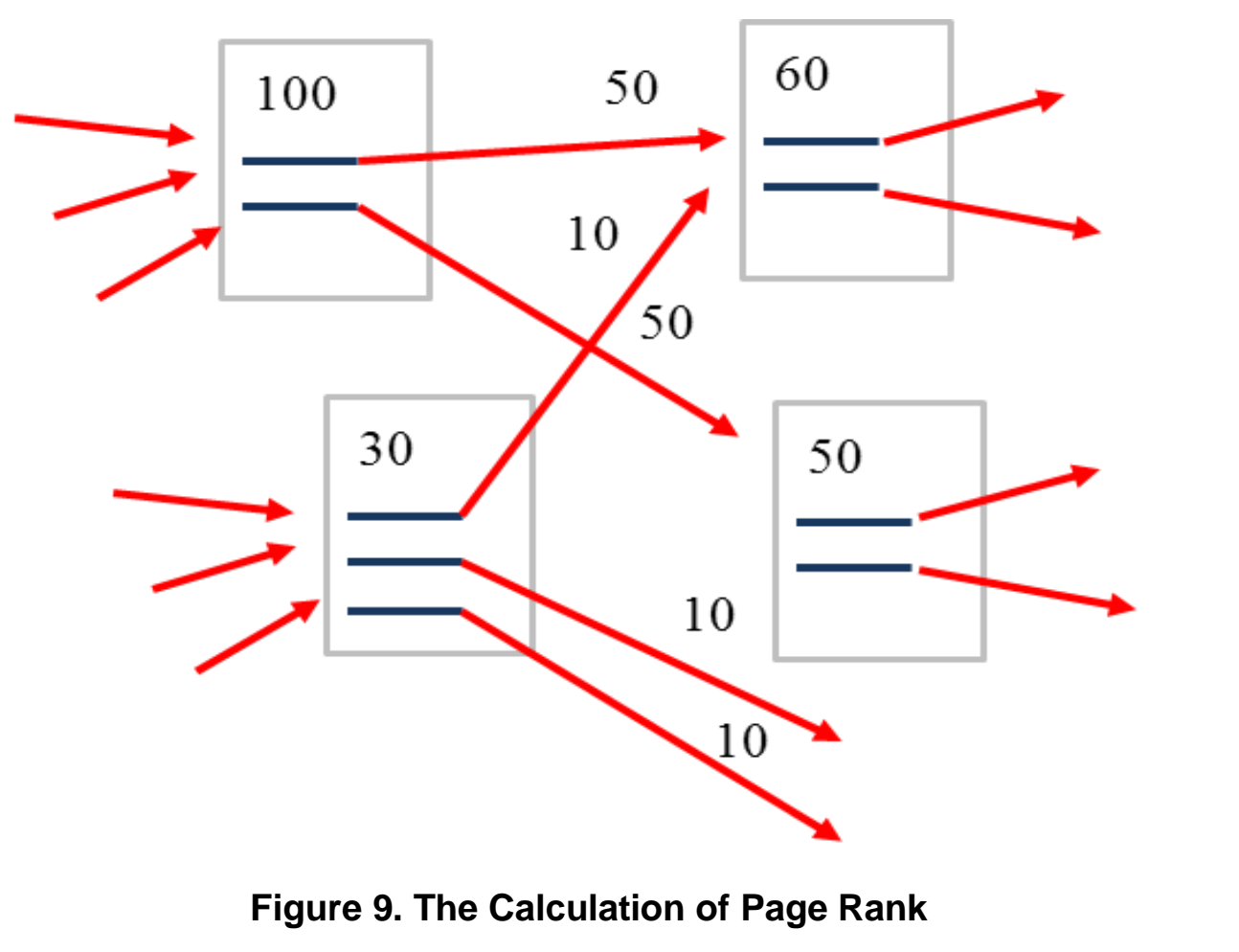

Each page has its own weight, andevalues which are the division of the weights by the number of links connected to each page are delivered. Equation (1) below is the expression for the page rank edalcylation.

$$
\ell P R(u)=\sum_{v_{\in} B_{u}} \frac{P R(v)}{L(v)}
$$

Let $\mathrm{Bu}$ is the set of pages pointing to page $\mathrm{u}, \mathrm{v}$ is element of $B u . P R(u)$ and $P R(v)$ are rank scores of page wand $v$. $L(2)$ is the number of links from $v$. Then rank score of page is determined by the set of links indicating the page and it is divided by the number of links from it and the divided yalues are added to each value of the linked pages.

Various methods using PageRank have been proposed. Taher proposed PageRank using Topic-Sensitive [13-14] and Wenpu Xing et al., proposed Weighted PageRank Algorithm [15]. Ropic-sensitive PageRank compute a set of scores of the importance of a page with respect to various topics. Weighted PageRank Algorithm assigns larger rank values to more important pages instead of dividing the rank value of a page evenly among its out-link pages. Each out-link page gets a value proportional to its popularity.

\section{PageRank based Bad Player Identification Scheme}

In this section, we propose our scheme to identify bad player which shows abnormal behavior including abusing and malicious attack on other players. Figure 10 shows our system architecture. Game database has players' game play data include play time, report time and link. Bad player rank has ranking of bad player and players, judgment point and bad player point. Decision system find bad player for restraint using normal distribution. In this paper, major features of the system are the 'Judgment Points' and the 'Bad Player Points'. The Judgment Points' are determined by the number of games played per each player and player's Bad Player Points and the Bad Player Points are determined who was reported as a bad player by the other player. 


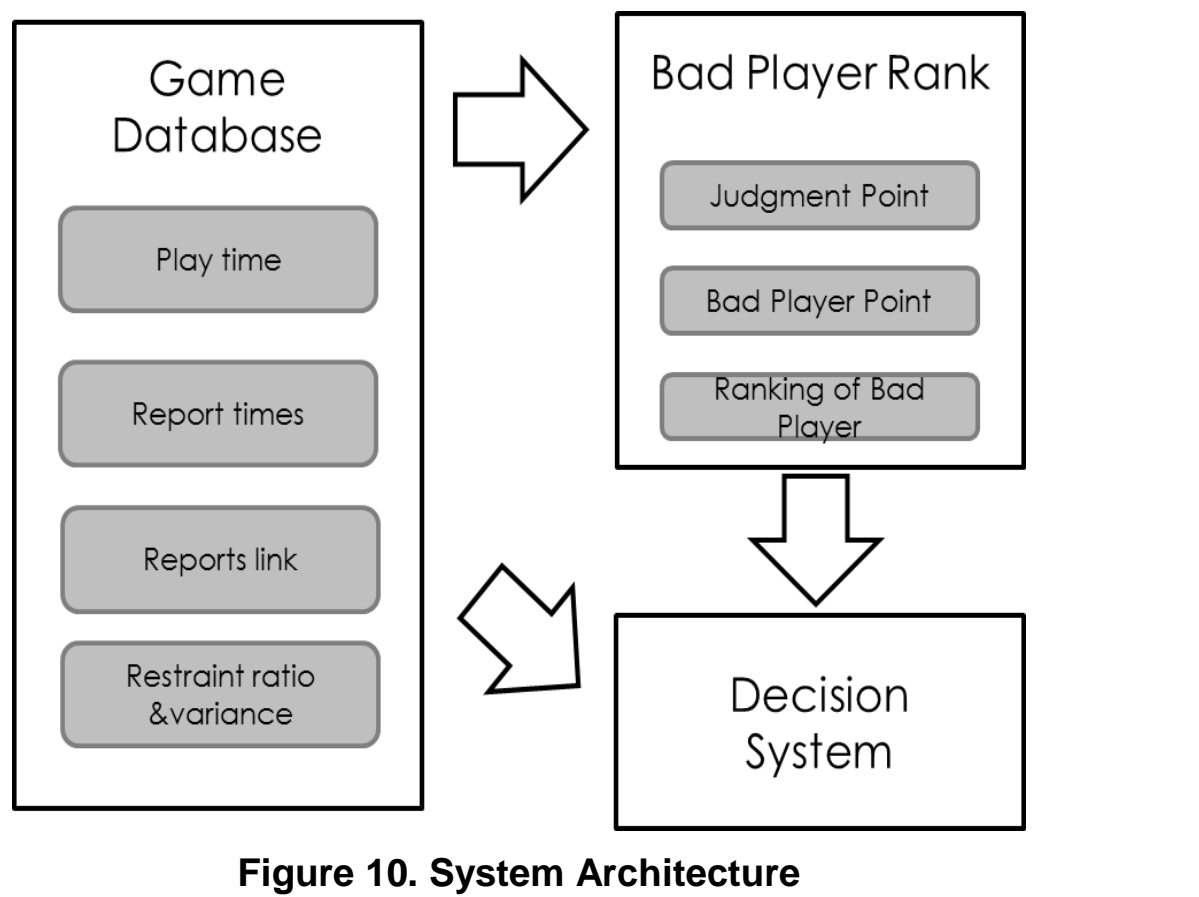

Figure 10. System Architecture

The pattern of overconfidence and under confidence observed in studies of intuitive judgment is explained by the hypothesis that people fogus on the strength or extremeness of the available evidence [16]. We use bad player rank for evidence and normal distribution for confidence.

\subsection{Evidence Accumulation for Bad Player Identification using PageRank} Algorithm

Player's Bad Player Points is areated by the Judgment Points of the players who reported player as bad playen and affects player's Judgment Points. Figure 11 shows how Bad Player Rank máde.

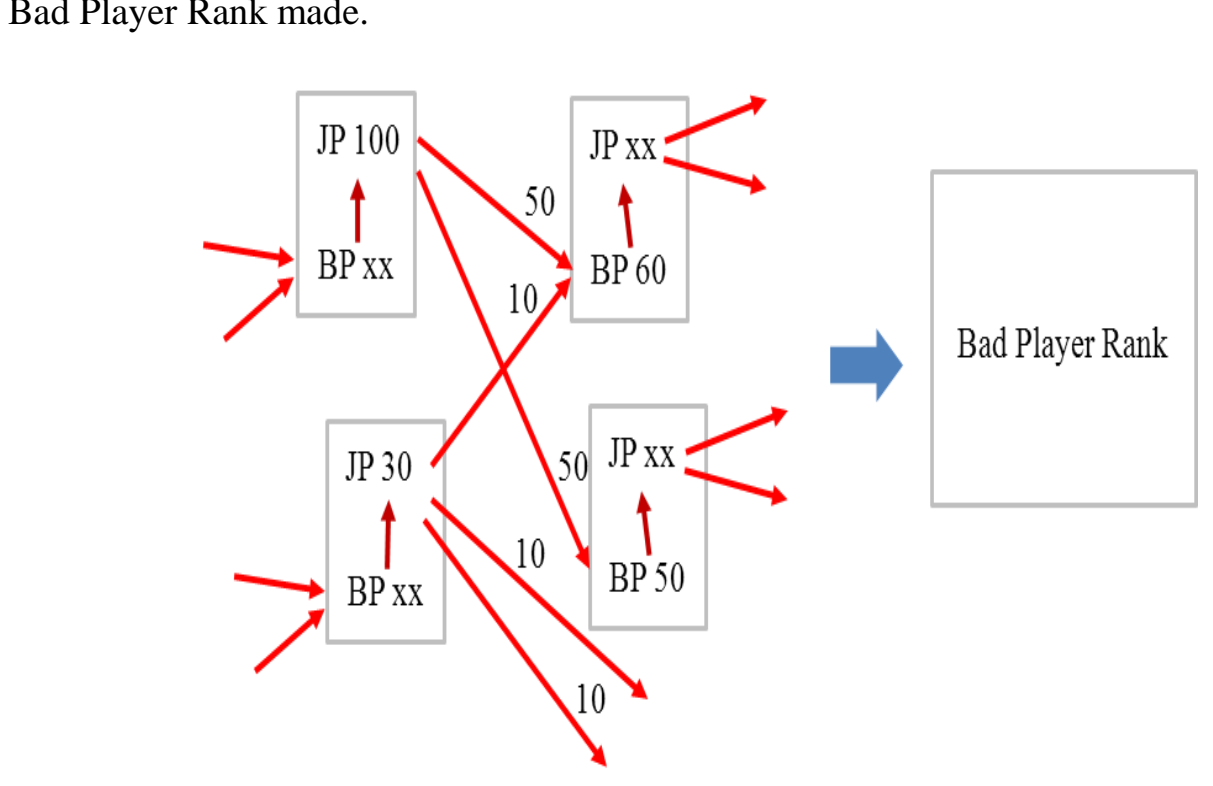

Figure 11. The Calculation of Bad Point and Judgment Point for Bad Player Rank 
The Judgment Points are configured by the average number of reporting and the time of game playing of the player and player's report time. Total Judgment Points of a player can be obtained by the equation (2) below. The Pt denotes the number of game playing time of the player, and the $\mathrm{Nr}$ denotes the average number of game playing time per one report of all players, and the $B P$ is the own Bad Player Points.

$$
J P=\frac{P t}{N r+B P}
$$

The $P t$ denotes the number of game playing of the player, and the $N r$ denotes the average number of game playing per one report of all players, and the $B P$ is the 'Bad Player Points' of each player. The 'Judgment Points' applicable to n players $(J P(n))$ who were reported by the player can be obtained from the following expression (3).

$$
J P(n)=\frac{P t}{N r+B P} \times \frac{1}{n} \quad(n>1)
$$

The value of Bad Player Points is the sum of JP divide report time of players reported other players as Bad Player. Let $R u$ denotes the set of other players Mo reported the player as Bad Player then the value of Bad Player Points of the player can be obtained by the following equation (4). $J P(k)$ denotes the value of JP divide report time of the other $k$ th player who reported the player as Bad Playen

$$
B P=\sum_{6 R^{2}} P(k)
$$

Number of line is player's repot for Judgment Pøints and who reported increase Bad Player Points also Judgment Points affected. The ranking of Bad Player can be determined by the value of Bad Player Points, and the player having higher ranking can be considered to have a bal career in the play of games. The value of Judgment Points of one player applicable to other players varies in accordance with the number of reporting and the value of Bad Points, thus it was presumed to support the reasonable decision making.

\subsection{Confidence Decision for) Bad Player Identification using Normal Distribution}

Our system selects the-top 30\% Bad Play Ranker and calculates who reported players' ratio of restraint with reported and its variance. We use total ratio of restraint with reported for confirmation of bad player based normal distribution. If mean is 0 and variance is 1 , the distribution is called the standard normal distribution or the unit normal distribution, and a random variable with that distribution is a standard normal deviate [17]. The normal distribution is the only absolutely continuous distribution all of whose probability distribution beyond the mean and variance are zero. It is also the continuous distribation with the maximum entropy for a given mean and variance. Equation (5) below is normal distribution function

$$
f(x, \mu, \sigma)=\frac{1}{\sigma \sqrt{2 \pi}} e^{-\frac{(x-\mu)^{2}}{2 \sigma^{2}}}
$$

This value is represented by a standard normal random variable. System determined by the sanctions if player's standard normal random variable is lower than bottom 5\% of the value (-1.65). Figure 12 shows our confidence decision distribution curve. 


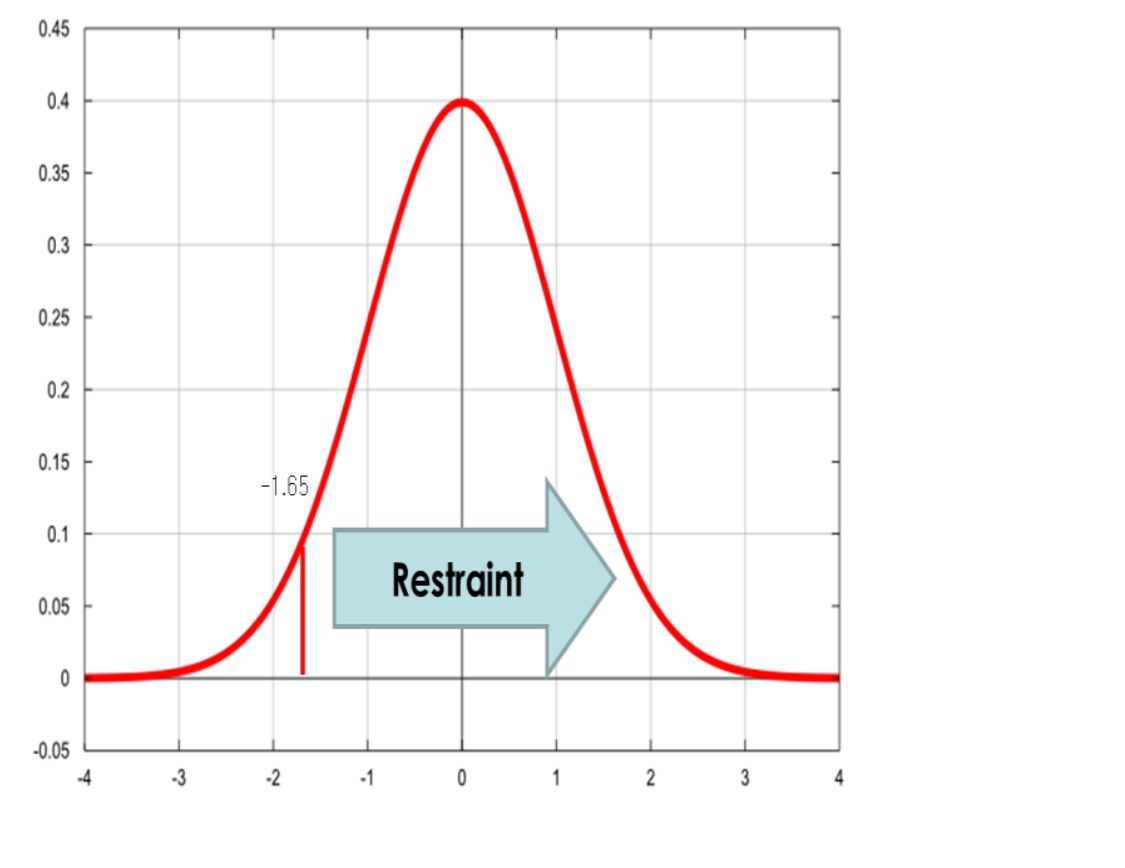

Figure 12. Confidence Decision Distripution Curve

Equation (6) below is standard normat random variable. $\mathrm{Z}$ is standard normal random variable and ${ }^{\wedge} \mathrm{P}$ is player's ratig of restraint with reported and $\mathrm{P}$ is total ratio of restraint with reported $\sigma$ is standard deviation of total rate.

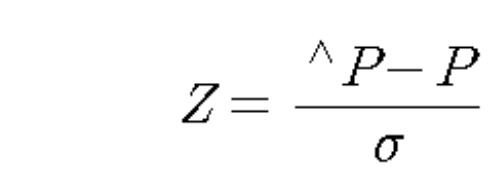

For example if total rate is $70 \%$ and standard deviation of total rate is $0.3 \%$ and player A and B has same Bad Player Points. Player A has $80 \%$ rate and player A has $60 \%$ rate. Standard normal random variable of player A is 1.82 , it is over than -1.65 . Player A receives restraint. But standard normal random variable of player B is -1.82 it is under than -1.65. Player B receives warning. If standard deviation of total rate is $0.8 \%$, standard normal random varjable of player $\mathrm{A}$ is 1.11 and standard normal random variable of player $B$ is -111 . Both variables are more than -1.65 and they receive restraint.

\section{Conclusions}

In this paper, we present a decision support system to find and identify bad players in MOBA games. The most concerned factor for normal players to get the victory in the MOBA game would be team strategy or team work along with personal skills. If such games ruined by bad players fail to attract further players to join in then the service providers would suffer a big loss. All the more, it would also be quite difficult to cope with every report generated over million times in a day. Thus it was assumed that the ranking system presented in this paper which exploits the value of Bad Player Points would be quite helpful to control bad players in such games. It was also presumed that the presented system can be applicable to other on-line games such as MMORPG. Our proposed system scheme has main advantage that it requires small computational efforts to identify bad players, because we utilize PageRank algorithm which shows efficient computation and information search capability. 


\section{References}

[1] N. Pobiedina, J. Neidhardt, M. D. C. C. Moreno, L. Grad-Gyenge and H. Werthner, "On Successful Team Formation: Statistical Analysis of a Multiplayer Online Game", 2013 IEEE 15th Conference, In Business Informatics (CBI), (2013), pp. 55-62.

[2] T. H. Kim, J. Y. Shim and S. W. Kim, "Proposition of PageRank based Decision Support System to Detect Bad Players in the MOBA Game", Advanced Science and Technology Letters, Games and Graphics, vol. 46, (2014), pp. 175-178.

[3] A. Rollings and E. Adams, "Fundamentals of Game Design", Prentice Hall, (2006).

[4] Riot Games, Inc. http://www.riotgames.com

[5] Valve Corporation. http://www.dota2.com.

[6] THE TRIBUNAL, http://na.leagueoflegends.com/tribunal/.

[7] A. Katal, M. Wazid and R. H. Goudar, "Big data: Issues, challenges, tools and Good practices", Contemporary Computing (IC3), 2013 Sixth International Conference on. IEEE, (2013).

[8] S. Kwon, M. Cha, K. Jung, W. Chen and Y. Wang, "Prominent Features of Rumor Propagation in Online Social Media”, ICDM 2013 (2013).

[9] B. Aditya Patel, M. Birla and U. Nair, "Addressing big data problem using Hadoop and Map Reduce", Engineering (NUiCONE), Nirma University International Conference, IEEE, (2012)

[10] G. Shafer, "A mathematical theory of evidence Princeton University'Press", (1976).

[11] S. Brin and L. Page, "The anatomy of a large-scale hypertextual Web search engine", Computer Networks and ISDN Systems, vol. 30, no. 1-7, (1998), pp. 107 117.

[12] L. Page, S. Brin, R. Motwani and T. Winograd, "The PageRank citation ranking. Bringing order to the web", (1999).

[13] T. H. Haveliwala, "Topic-Sensitive PageRank", WWW 02 Proceedings of the 11th international conference on World Wide Web", (2002), pp. 517,526

[14] T. H. Haveliwala, "Topic-Sensitive PagęRank. A Context-Sensitive Ranking Algorithm for Web Search", IEEE Transactions on Knowledge and Data Engineering, vol. 15, no. 4, (2003), pp. 784-796.

[15] W. Xing and A. Ghorbani, "Weighted PageRank Algorthm", Proceedings of the Second Annual Conference on Communication Networks and Services Research, (2004).

[16] D. Griffin and A. Tversky, "The freghing of evidence and the determinants of confidence", Cognitive psychology, vol. 24, no. 3, (1992), pp. 411-435.

[17] G. Casella and R. L. Berger, "Statistical Inference(2nd ed.)", Duxbury, (2001).
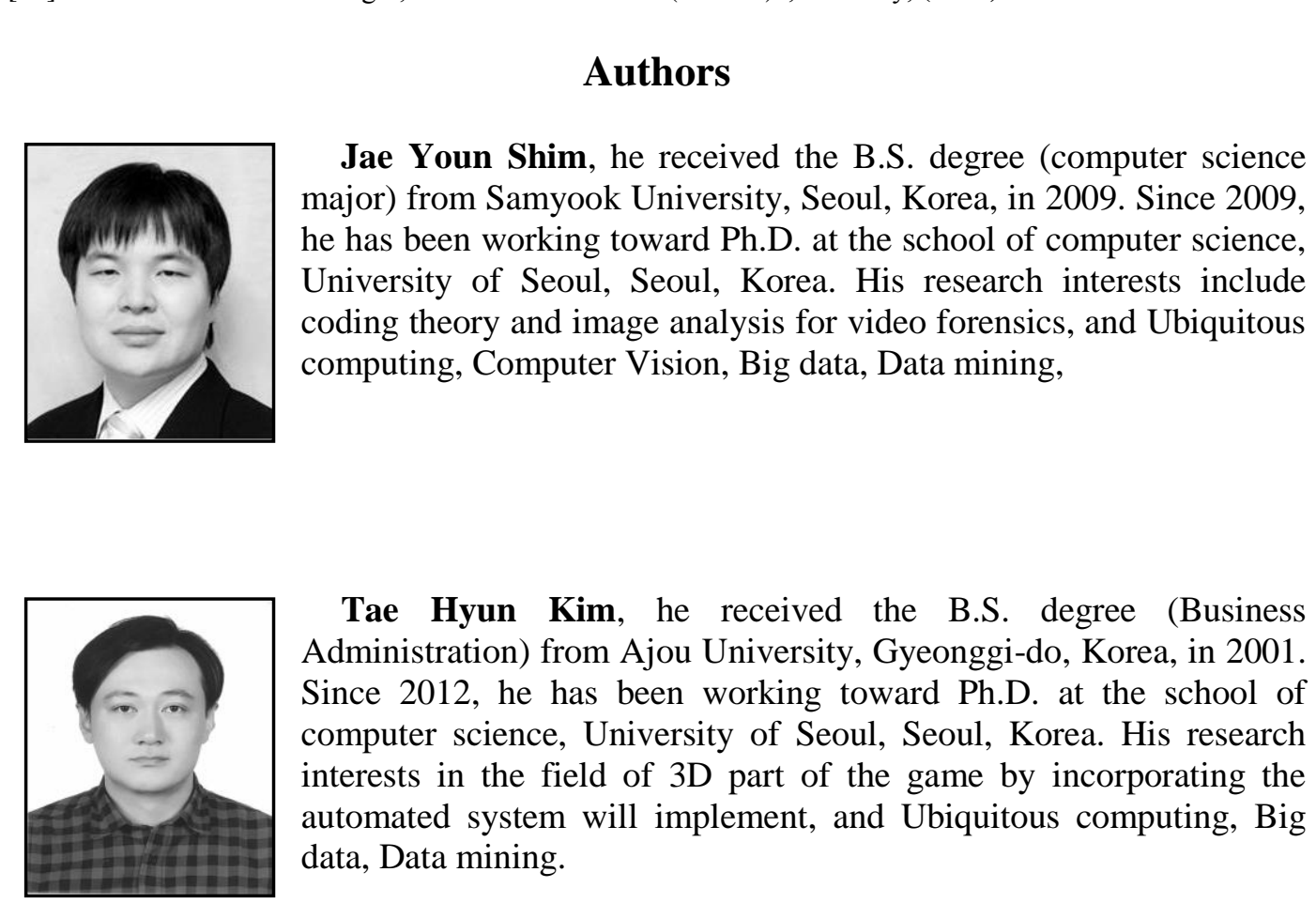

Tae Hyun Kim, he received the B.S. degree (Business Administration) from Ajou University, Gyeonggi-do, Korea, in 2001. Since 2012, he has been working toward Ph.D. at the school of computer science, University of Seoul, Seoul, Korea. His research interests in the field of 3D part of the game by incorporating the automated system will implement, and Ubiquitous computing, Big data, Data mining. 


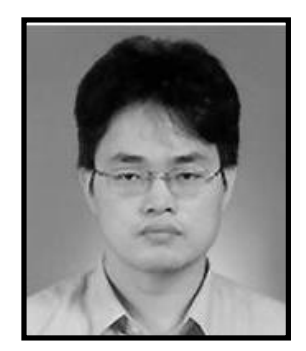

Seong-Whan Kim, he received his Ph.D. degree in EECS from KAIST, Taejon in 1999. He served as a software team leader in LG Electronics for IMT-2000 infrastructure division from 19972000 , and worked as a senior software engineer in wireless LAN infrastructure design team of Cisco Systems Inc. from 2000 to 2001. He worked as a visiting professor for University of North Carolina (2005-2006) and University of Illinois, UrbanaChampaign (2012-2013), respectively. He is currently a full professor at the school of computer science, University of Seoul, Seoul, Korea. His research interests include mobile communication systems, computational approach to visual arts, mathematical modeling of human visual system, and its application to multimedia coding/restoration.

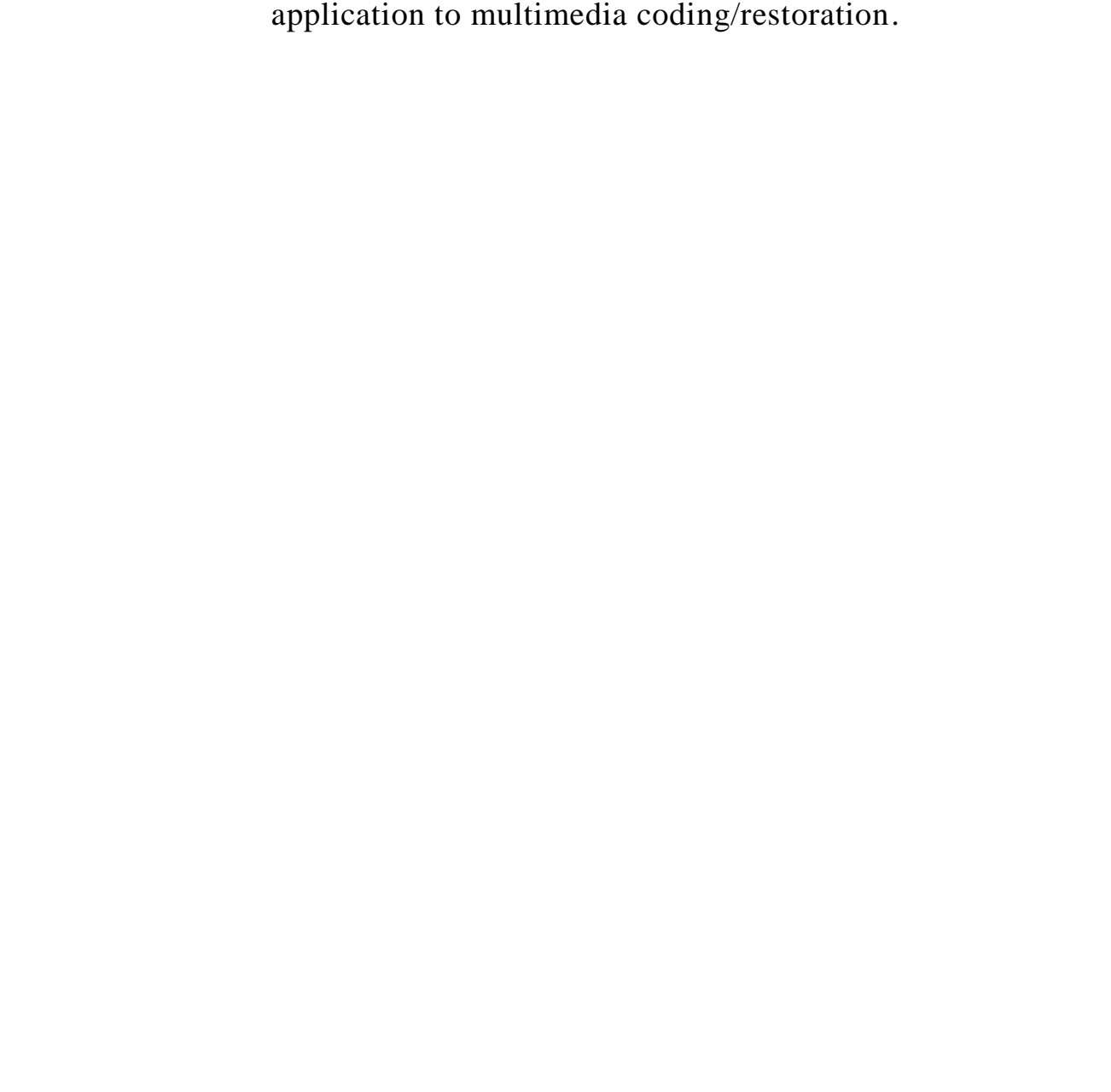


International Journal of Multimedia and Ubiquitous Engineering

Vol.9, No.8 (2014)

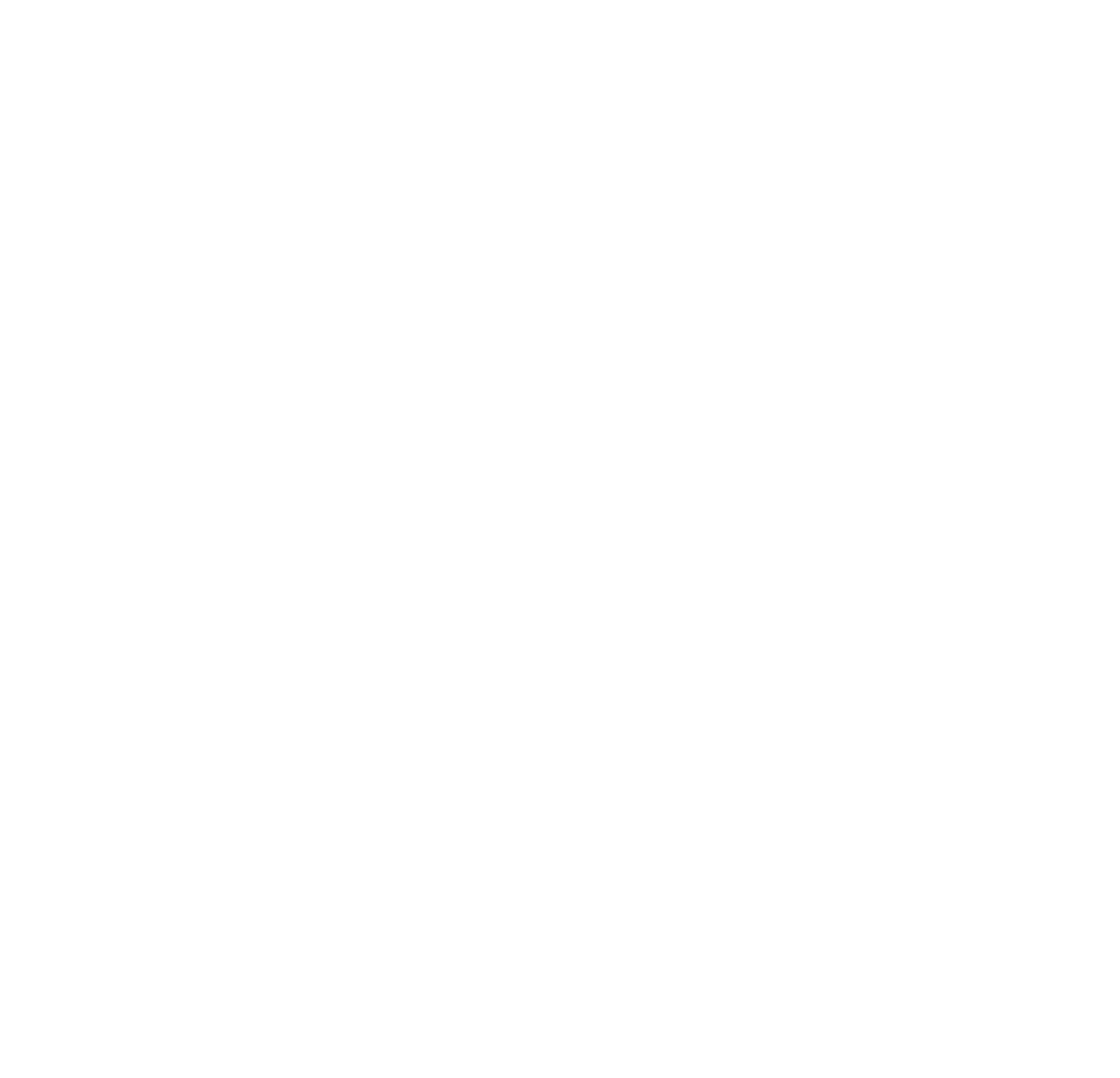

